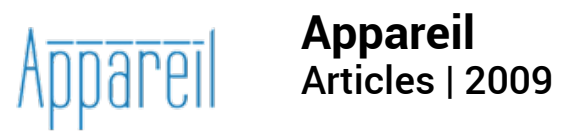

\title{
Le cinéma, de Walter Benjamin à Pier Paolo Pasolini
}

Alain Naze

\section{(2) OpenEdition}

Journals

Édition électronique

URL : http://journals.openedition.org/appareil/875

DOI : 10.4000/appareil.875

ISSN : 2101-0714

Éditeur

MSH Paris Nord

Référence électronique

Alain Naze, «Le cinéma, de Walter Benjamin à Pier Paolo Pasolini », Appareil [En ligne], Articles, mis en ligne le 04 novembre 2009, consulté le 31 juillet 2020. URL : http://journals.openedition.org/appareil/ 875 ; DOI : https://doi.org/10.4000/appareil.875

Ce document a été généré automatiquement le 31 juillet 2020.

\section{(c) (i) (9)}

Appareil est mis à disposition selon les termes de la Licence Creative Commons Attribution - Pas d'Utilisation Commerciale - Pas de Modification 4.0 International. 


\title{
Le cinéma, de Walter Benjamin à Pier Paolo Pasolini
}

\author{
Alain Naze
}

Une mise en écho des œuvres et démarches respectives de Benjamin et de Pasolini peut trouver son point de départ du côté d'une certaine pensée de l'histoire, qui, tout en n'étant pas romantique, fait du passé « ce qui peut sauver la révolution », selon la belle formule pasolinienne concluant le film La rabbia; on pourrait évoquer alors, dans cette direction, le marxisme si peu orthodoxe de l'un et de l'autre ; mais le parallèle pourrait tout autant s'esquisser à partir de la conception du langage que tous deux développent, conception intempestive dans les deux cas, pour l'accent qu'elle fait porter sur la question de l'origine du langage, là où la science linguistique $\mathrm{du} \mathrm{xx}^{\mathrm{e}}$ siècle avait pourtant frappé d'interdit ce type de questionnement, pour la dimension métaphysique dont elle serait porteuse; et alors on pourrait évoquer les accents prophétiques qu'a pu emprunter la parole de l'un ou de l'autre - et la liste de telles correspondances entre Benjamin et Pasolini pourrait être aisément poursuivie, faisant apparaître ainsi une résonance, significative en elle-même. Mais ici, c'est autour de la question du cinéma que la mise en relation des deux œuvres va plus spécifiquement nous intéresser, du moins à travers quelques-uns de ses aspects. Bien sûr, l'interrogation de Benjamin relative à la question du cinéma reconduit à toute sa réflexion sur l'œuvre d'art. Rappelons-nous qu'en ce qui concerne la photographie, il se demandait moins s'il s'agissait encore d'œuvre d'art, qu'il ne s'interrogeait sur les effets que l'apparition de la photo avait pu avoir sur l'art lui-même - et cette remarque peut évidemment être reportée, à l'identique, dans le champ de l'apparition du cinéma. Mais ici, donc, en restreignant le champ de la question, on va s'interroger sur un certain rapport que le cinéma entretiendrait, du moins selon l'optique de nos deux auteurs, entre la narration et le choc. Il ne s'agit bien évidemment pas de faire du cinéma l'appareil par excellence de la narration, mais seulement d'envisager le fait que, si une dimension narrative est nécessairement présente dans tout film (ce que soutient Pasolini, on le verra), la spécificité du cinéma, dans le cadre d'une narrativité peut-être indépassable, pourrait bien consister en la provocation d'un certain type de choc, ou de télescopage qui, par la rupture ainsi introduite dans un continuum temporel, ne serait pas sans rapport avec 
cet "arrêt dialectique " que Benjamin désigne comme constitutif de "l'image dialectique», pensée comme un certain rapport entre «l'Autrefois» et le « Maintenant ».

2 Si la question de la narration paraît centrale dans le rapport au cinéma qu'entretiennent Benjamin et Pasolini, c'est essentiellement en ce que le cinéma, forme artistique moderne par excellence, semble susceptible de prendre le relais des formes traditionnelles de narration. Par conséquent, il ne s'agit pas là du tout d'envisager un cinéma qui aurait à se plier, par exemple, aux conditions d'énonciation narrative propres à la littérature, mais de considérer que si la narration survit dans le cinéma, alors se trouve ainsi dessinés les contours d'une manière spécifiquement moderne de raconter. Là encore : au lieu de nous demander si le cinéma relève bien de l'ordre de la narration, demandons-nous plutôt comment le cinéma transforme la narration. D'ailleurs, l'ultime livre de Pasolini (Pétrole), qu'il présente essentiellement comme une «forme », emprunte fréquemment les voies d'une écriture de type cinématographique, ce qui montre bien que si le cinéma a partie liée à la narration, cela ne signifie pas nécessairement qu'il doive obéir aux exigences hétéronomes de la littérature, puisqu'il serait lui-même capable d'influer sur les formes littéraires de la narration.

sait que Benjamin s'est particulièrement intéressé aux formes traditionnelles de l'art de raconter, et le lien qu'il établit entre la narration et les formes sociales montre bien que l'univers en lequel elle restait possible ayant disparu, si quelque chose comme de la narration subsiste, ce ne peut être que sous des modalités très différentes. Leskov tracerait ainsi la figure du dernier narrateur traditionnel, celle d'un monde disparaissant, mais on se tromperait en concluant pour autant à la mort de la narration, Benjamin soutenant bien plutôt que « [1]a narration, elle, demeurera. Mais pas sous sa forme "éternelle", dans sa chaleur familière et souveraine ; plutôt sous des formes inédites, audacieuses, desquelles nous ne savons encore rien ${ }^{1}{ }^{1}$. Si, de cette façon, le lien n'est pas positivement établi entre cinéma et narration, la prudence de Benjamin rend au moins son propos accueillant à l'idée selon laquelle le cinéma constituerait peut-être une des sources inédites de formes non traditionnelles de narration. L'attrait de Benjamin pour Eisenstein et peut-être surtout, même s'il ne paraît jamais l'évoquer directement, pour Vertov, semble pourtant nous orienter vers un cinéma de montage avant tout, c'est-à-dire pas directement narratif, et même, dans le cas de Vertov, résolument opposé à un cinéma de récit. Cette contradiction semble susceptible d'être dépassée, si l'on s'appuie sur l'idée pasolinienne selon laquelle il n'y a pas de cinéma qui ne soit narratif, au moins malgré lui. Pasolini dit en effet continuer à "croire au cinéma qui raconte», jugeant qu'un film ne peut pas ne pas raconter quelque chose : face aux tentatives de nombreux réalisateurs cherchant à obtenir que " même au cinéma "il ne se passe rien" "², s'alignant en cela "sur le nouveau roman » et sur certaines avant-gardes qui parlent d' "anti-roman », Pasolini, lui, déclare ne pas y croire, " parce que toute forme d'art ne fait qu'évoquer la réalité, et rien d'autre, et dans la réalité il se passe toujours quelque chose, comme le temps passe, ou du moins donne-t-il l'impression de passer : et ceci est l'illusion de notre vie ${ }^{3}$. La question qui se pose ici est de chercher à comprendre comment Pasolini en arrive à cette conclusion, c'est-à-dire à l'idée d'une impossibilité d'un cinéma non narratif, tout en opposant un " cinéma de prose » à un « cinéma de poésie ".

4 En fait, il faut bien saisir que par l'expression de "cinéma de poésie », il ne désignait qu'une « notion abstraite », et ne cherchait à définir par là ni « la forme principale du 
cinéma moderne ", ni celle qu'il aurait lui-même adoptée, puisque tout au contraire, il visait ainsi à cerner une notion "valable pour tous les temps", et qui aurait même dominé avec «le cinéma des origines »4. Par conséquent, lorsqu'il parlait de « cinéma de poésie ", loin de considérer qu'il désignait ainsi un cinéma absolument non narratif, Pasolini visait d'abord des films relevant d'une "poésie narrative ", à l'image de cet Homme d'Aran, de Robert Flaherty, à propos duquel il écrit qu'on se trouve ici face à " une idée du montage assujetti à une technique narrative du cinéma de poésie »5. Et en effet, si le personnage central du film est sans doute l'île irlandaise elle-même et la population qui l'habite, précisément contre vents et marées - et cela pourrait en effet nous conduire vers un cinéma non narratif, consistant seulement à enregistrer ce qui est, à la manière de Vertov éventuellement - il n'en reste pas moins (et ce fut d'ailleurs l'objet des critiques de la part des tenants du documentaire au sens strict) que le film de Flaherty est monté de façon à faire apparaître comme une épopée les activités de travail qui conditionnent la survie sur ces rochers désertiques - qu'on pense seulement au caractère haletant des scènes relatives à la pêche au requin-pèlerin, lors desquelles naît une inquiétude pour les personnages eux-mêmes (à l'unisson de Michaël et sa mère), et plus largement pour l'issue de l'épisode. Autrement dit, si le cinéma de poésie se distingue bien d'un cinéma de narration, «[1]a différence ressorti[r]ait à la technique : plutôt que la technique narrative du roman, de Flaubert ou de Joyce, la technique narrative de la poésie $»^{6}$, et c'est en cela que la sorte de poème épique de Flaherty peut être rejoint dans son genre par «les histoires parisiennes d'intérieurs, chambres à coucher ou bars, de Godard, [qui] sont montées avec une technique narrative typique de la poésie $»^{7}$. Il existe donc bien une certaine porosité entre les deux formes typiques de cinéma répertoriées par Pasolini, et cette remarque est utile pour saisir les passerelles entre "pseudo-récit" des films relevant d'un cinéma de poésie, et "poésie interne » propre à certains films narratifs, ni l'un ni l'autre ne relevant, en tout cas, d'un « cinéma de poésie non narrative ", c'est-à-dire de " poésiepoésie $»^{8}$.

Pour définir de la manière la plus simple possible le «cinéma de poésie », Pasolini propose de reprendre la formule que les spécialistes utilisent: "Faire sentir la caméra »; les films relevant d'un cinéma de poésie prendrait donc le contre-pied de la formule valant pour les "cinéastes sages ", jusqu'au début des années soixante : « Ne pas faire sentir la caméra $»^{9}$. Dans ces conditions, Pasolini se doit de reconnaître que s'il $\mathrm{y}$ avait une poésie propre aux "grands poèmes cinématographiques, de Charlot à Mizoguchi et à Bergman $»^{10}$ (et qu'on retrouverait, à sa manière, dans L'homme d'Aran), du moins n'obéissait-elle pas aux canons définissant le cinéma de poésie - on n'y sentait pas la caméra. C'est donc bien que la poésie de ces films se situait «ailleurs que dans le langage en tant que technique du langage ", autrement dit, qu'elle n'en passait pas par l'adoption d'un "langage spécifiquement poétique», et que par conséquent, «ce n'étaient pas des poèmes, mais des récits: le cinéma classique a été et reste narratif: sa langue est celle de la prose "; on se trouverait donc face à une poésie " interne ", à l'image de celle qui traverserait " les récits de Tchekhov ou de Melville " ${ }^{11}$. Pasolini réservera donc le terme de "pseudo-récit » aux films relevant du cinéma de poésie : « [1]a formation d'une "langue de la poésie cinématographique" implique donc la possibilité de faire, au contraire [au contraire des récits propres au cinéma classique], des pseudo-récits, écrits dans la langue de la poésie: la possibilité d'une prose d'art, d'une série de pages lyriques, dont la subjectivité est assurée par l'usage, servant de prétexte, de la "subjective indirecte libre"; et dont le véritable protagoniste 
est le style ${\aleph^{12}}^{12}$. Ainsi, s'il n'y a pas de cinéma non narratif, ce qui explique que Pasolini parle de "pseudo-récits" pour les films relevant d'un "cinéma de poésie ", c'est d'abord parce qu'il n'y a pas de cinéma usant d'une langue lyrique. Mais si l'impossibilité, au moins actuelle, d'une poésie non narrative au cinéma implique que le cinéma de poésie relève de la prose (poétique), on peut dire aussi, en sens inverse, qu'il existe également une poésie « interne " propre au cinéma de prose, ou peut-être plutôt qu'il existait une telle poésie interne - le passage à l'imparfait se justifiant par le fait que Pasolini décrive ces récits à partir de conditions de possibilité qui ne semblent plus à notre portée : «[1]e fait que l'on n'y sentît pas la caméra signifiait que la langue adhérait aux significations, en se mettant à leur service: elle était transparente jusqu'à la perfection : elle ne se superposait pas aux faits, en les violentant à travers les folles déterminations sémantiques que l'on doit à sa présence comme conscience technicostylistique continue $\aleph^{13}$. Pour comprendre ces mots de Pasolini, il ne faut pas oublier que pour lui, le cinéma est expression de la réalité par la réalité, et que dans ces conditions, les modifications de la réalité elle-même (c'est-à-dire tout autant des ressources du langage cinématographique) rejaillissent sur les possibilités d'expression propres au cinéma. Semblerait ainsi révolue l'époque d'une certaine transparence de la langue utilisée, où il était encore possible de filmer sans faire se déplacer la caméra, tout en obtenant des effets remarquables (Pasolini évoque ici Les lumières de la ville, à travers "le comique stupéfiant du ballet de Charlot", boxant face à un champion beaucoup plus fort que lui, comique obtenu à partir d'une caméra restant immobile, prenant « un plan d'ensemble quelconque $»^{14}$ ). Par ce biais, on retrouve, cette fois dans le cadre du cinéma, une remarque de Benjamin relative à la littérature, plus précisément à l'impossibilité moderne de raconter selon les canons de la narration traditionnelle : «[...] s'il n'y a plus de bonnes histoires à écouter, c'est aussi que les choses ne durent plus de la bonne manière $»^{15}$. Ainsi, le cinéma connaîtrait peut-être, en accéléré, une évolution qui ne serait pas sans rapport avec la littérature : tout comme il n'est plus possible aujourd'hui d'écrire, simplement: "La marquise sortit à cinq heures ", la transparence du narrateur ayant vécu, de même, le cinéaste ne pourrait plus filmer en toute transparence la marquise, sortant en effet à cinq heures. Car ces évolutions, en apparence essentiellement stylistiques, signifieraient d'abord une transformation de la réalité elle-même: de la même manière que Benjamin indique bien que les grandes figures (nomades et sédentaires) de la narration ont disparu avec l'avènement du monde moderne, et du roman bourgeois, Pasolini indique pour sa part que le caractère expressif de la réalité tendant à se dissoudre (dans le cadre d'une réalité devenue technique, pour les objets, et, plus tard, sous les coups de «l'homologation consumériste " pour les corps, les voix, etc.), il est devenu impossible de réaliser des poèmes cinématographiques à la manière de Bergman, Dreyer, ou Mizoguchi, qui supposaient le caractère " poétisable » de la réalité. Pasolini, à cet égard, écrit : « [...] il y a quelque chose dans la vie moderne qui n'est plus poétisable [...] [o]u qui n'est poétisable qu'une seule fois ", à l'image de l'usine filmée dans Les temps modernes, vis-àvis de laquelle Charlot pouvait rester "expressif», en se détachant sur le fond de fonctionnalité propre à la technique - alors qu'aujourd'hui, selon les mots mêmes de Pasolini, « la vie à l'usine tend à s'imposer comme modèle fondamental et même unique de toute la vie ", ce qui implique que le personnage de Charlot serait à présent intégré à la réalité elle-même, laquelle ne serait plus capable de sécréter quoi que ce soit échappant à la fonctionnalité, et donc surtout pas un équivalent de Charlot, ce personnage qui était «tout expressivité ${ }^{16}$. Soit, donc, la réalité n'est plus poétisable, 
soit elle ne l'est qu'une seule fois. Par conséquent, le cinéma lui-même participerait à cette technicisation par laquelle la réalité ne pourrait plus faire l'objet d'un poème cinématographique : la réalité ayant elle-même intégré les films réalisés (au sens où ces films font partie de cette réalité, et conditionnent en partie la manière dont on la perçoit), c'est à un appareillage redoublé de la réalité auquel on assiste ici (comme l'appareil narratif spécifiquement littéraire avait constitué un appareillage antérieur), et c'est donc à un retour de la médiation qu'on doit conclure (là où Pasolini visait, par le recours au cinéma, à supprimer une médiation entre lui et la réalité), dans le sens où un signe cinématographique peut désormais renvoyer aussi à un autre signe cinématographique - ainsi, les films à la poésie « interne » dont parle Pasolini auraient connu une situation idyllique, qui aurait proprement été celle de la mise en place de l'appareillage cinématographique appliqué à une réalité qui en était encore vierge. C'est donc cette intégration de la technique cinématographique à la réalité que Pasolini veut rendre visible, afin de déjouer les mystifications de la technique : « Les techniques audio-visuelles constituent désormais une grande part de notre monde, c'est-à-dire du monde du néo-capitalisme technique qui avance, et qui tend justement à rendre ses techniques a-idéologiques et ontologiques; à les rendre tacites et absolues; à en faire des habitudes ; à en faire des formes religieuses. Nous sommes des humanistes laïques, ou au moins des platoniciens non misologues : c'est pourquoi nous devons nous battre pour démystifier l' "innocence de la technique", jusqu'au bout $\aleph^{17}$. On retrouve ici une préoccupation qui sera aussi celle de Benjamin, à travers son opposition entre une " première technique » (visant essentiellement à une maîtrise des forces naturelles) et une "seconde technique » (envisagée comme plus émancipatrice, précisément en sa tendance à s'écarter d'une forme d'imitation de la nature), englobant bien évidemment le cinéma ; Benjamin s'interroge en effet sur les capacités effectivement émancipatrices de cette seconde technique : «En face de cette seconde nature, l'homme, qui l'inventa mais qui, depuis longtemps, n'en est plus le maître, a besoin d'un apprentissage analogue à celui dont il avait besoin en face de la première nature. Une fois de plus, l'art est au service de cet apprentissage. Et notamment le cinéma. [...] Faire de l'immense appareillage technique de notre époque l'objet de l'innervation humaine, telle est la tâche historique au service de laquelle le cinéma trouve son sens véritable " 18. On sait que Benjamin définit cette tâche libératrice propre au cinéma comme le renversement de la testabilité (passive) en maîtrise (active), autrement dit, un renversement du sens de la caméra qui, d'appareil d'enregistrement devient ce qui rend le test lui-même exposable - loin de tout pas en arrière, l'émancipation se gagnerait donc à l'intérieur même de la seconde technique. C'est précisément en ce sens que Pasolini, tout en s'interrogeant à voix haute (« Devrai-je rendre compte, dans la vallée de Josaphat, de la faiblesse de ma conscience face aux séductions, qui s'identifient, de la technique et du mythe ? ${\aleph^{19}}^{9}$, poursuivra jusqu'à sa mort ses expérimentations au sein du médium cinématographique, tout en continuant à l'interroger - mais de l'intérieur - quant à sa capacité politique de résistance.

6 L'ultime film de Pasolini, Salo ou les cent vingt journées de Sodome, constitue, par excellence, un film sur la capacité du cinéma à demeurer un moyen politique efficace, malgré (et du sein même de) son autoréférentialité, ici pleinement assumée. La proximité avec Benjamin se révèle encore ici, en ce que le questionnement de Salo, au fond, consiste à rechercher la brèche susceptible de briser la temporalité homogène du présent, d'interrompre la continuité du temps de la catastrophe ; et si cette proximité 
est encore celle qui les relie au cinéma en tant que tel, c'est que la réponse de Pasolini sera de part en part cinématographique.

Dans Pétrole, Pasolini utilise une métaphore du cinéma, à travers l'idée du personnage central du "roman», Carlo, en caméraman, dont les visions (dans le viseur de l'objectif) sont expliquées et commentées par trois petits dieux présentés comme l'élément rationnel du dispositif. Par là, Pasolini illustre son idée selon laquelle, quelle que soit la visée du réalisateur, en termes de signification, les images d'un film débordent toujours cette intention volontariste et consciente, en ce que c'est la réalité elle-même qui se présente au spectateur, avec son poids de simple présence, malgré tout, brute : les éléments d'un film provenant de la réalité elle-même, «les images sont toujours concrètes, jamais abstraites », et donc, si le cinéma peut atteindre le stade de la « parabole ", à aucun moment il ne peut se faire " expression conceptuelle directe ", la «matérialité onirique » de ses images s'y opposant formellement ${ }^{20}$ - irrationalité cinématographique que le dispositif de Pétrole souligne, tout en y apportant un remède, mais spécifiquement littéraire. En revanche, la faille dans la manière de filmer, que le roman évoque, trouve, elle, un prolongement cinématographique dans l'œuvre de Pasolini. C'est le passage de Pétrole en lequel Carlo, filmant la réalité d'une Italie de l'époque succédant à ce que Pasolini appelle le "génocide culturel », va se trouver face à un "déphasage ", une sorte d'erreur technique, qui aura pour effet de substituer à la hideuse réalité contemporaine (constituée d' «immeubles neufs») un monde immémorial, à travers «des masures et les piliers d'un Aqueduc» de "ce qui fut autrefois une grande métropole plébéienne $»^{21}$. Cinématographiquement, cette erreur technique prendra la forme d'une manière de filmer contrevenant parfaitement aux règles de la narration, lorsque, en particulier dans Les mille et une nuits, on se trouve face à des figurants du Tiers Monde qui fixent du regard la caméra. Un anachronisme comparable à celui qu'évoque le roman se produit alors, les corps plébéiens crevant ainsi littéralement l'écran (du moderne cinéma), en opérant une rupture dans la continuité temporelle de la narration, enrayant le récit par la fixation ainsi effectuée. On se trouve ici face à un équivalent cinématographique de ce que Benjamin appelle "image dialectique», c'est-à-dire, en l'occurrence, face à un télescopage entre la modernité du médium cinématographique et «l'Autrefois " propre à des corps sans âge, rencontre susceptible de faire briller, un instant, une réalité disparue, précisément à la faveur de "l'arrêt dialectique " ainsi opéré. L'homogénéité du présent a donc su être percée par le surgissement d'une extériorité. C'est, dans une certaine mesure, le même problème qui se pose dans Salo ou les 120 journées de Sodome, à ceci près qu'à la différence des films de la Trilogie de la vie, Pasolini a renoncé à chercher des corps plébéiens susceptibles de briser la triste répétition des corps soumis à l'homologation consumériste, par où la sortie hors du dispositif carcéral de Salo devra nécessairement emprunter d'autres voies.

De ce dernier film de Pasolini, on peut dire qu'il constitue une réflexion théorique en acte sur le cinéma, en ce qu'il pose la question de la place du spectateur comme voyeur (à la fois parce que nous regardons des actes de torture, des crimes, sans nous y opposer, et également parce que le film lui-même représente aussi les bourreaux qui, tour à tour, seront les spectateurs jouissant des crimes qui se commettent dans la cour), mais aussi celle de la complicité du réalisateur avec ce qu'il montre, quelles que puissent être ses intentions de dénonciation (le poids de réalité des images qu'on évoquait tout à l'heure, s'imposant à l'écran, se trouve ici métaphorisé à travers les paroles littéraires de Pasolini lui-même, qui seront, parce qu'elles sont prononcées par 
les bourreaux du film, compromises avec celles de tous les autres auteurs cités). C'est bien l'enfermement qui caractérise ce film, formellement et aussi du point de vue de l'histoire, et l'on est bien ici - si l'on ajoute l'autoréférentialité de l'œuvre, empêchant tout surgissement d'un dehors - dans ce que Deleuze a pu appeler, parlant de Salo, « un pur théorème mort, un théorème de la mort» (en opposition à Théorème, qui constituerait, lui, " un problème vivant ", à travers la solution provenant du dehors, l'hôte $)^{22}$. Que ce film dût être mal compris, c'est ce que Pasolini prévoyait, qui comptait défendre son film, au moment de sa sortie, comme on mène une "guérilla " - c'est qu'en effet, le risque est grand de l'indistinction entre le film lui-même et ce qu'il dénonce. Dans ces conditions, et Pasolini connaissant la puissance suggestive des images, la faille se doit d'être cherchée à une hauteur comparable, c'est-à-dire sur un terrain strictement cinématographique, une signification de parabole ne pouvant destituer tout à fait ce que des images ont constitué, à un niveau autrement profond. Dès lors, le sens que Pasolini confère à ce film, et qui en fait une dénonciation de l'univers consumériste (du "nouveau fascisme»), à travers une représentation empruntant les oripeaux du nazi-fascisme, ne peut valoir qu'à titre indicatif, quelque chose de plus puissant devant agir dans ce film, sans quoi il serait digéré, absorbé par ce qu'il était censé combattre.

9 Or, si l'on considère Salo en son ensemble, on peut s'apercevoir qu'il se caractérise, notamment, par la présence de quelques moments de pur cinéma, c'est-à-dire de cinéma muet, que Pasolini, d'ailleurs, affirme préférer au parlant. Et il se trouve que ces moments sont particulièrement expressifs, que l'on songe à cette tentative de fuite d'un prisonnier, lors de son transfert, geste de rébellion intervenant dans un moment muet du film, seulement troué par les rafales qui abattront le fugitif; ou qu'on envisage ce point levé du milicien ayant désobéi au règlement en couchant avec une servante, et qui se défait de son statut de collaborateur à travers cette nudité, moment muet là encore, auquel il est à nouveau mis fin par les coups de feu des bourreaux, l'exécutant ainsi. Mais c'est finalement toute la dernière partie du film (le "Cercle du sang ») qui s'avère également être quasiment muette, silence au sein duquel a lieu la défenestration suicidaire de la dernière narratrice (pianiste bien plutôt, puisque presque muette), après avoir vu par la fenêtre quelque chose que le spectateur ignore (sans doute des crimes dans la cour), et silence auquel met fin le bruit mat du corps au moment où, on l'imagine sans le voir, il touche le sol. Ces trois moments pourraient être enregistrés comme des moments de résistance, mais tous s'achevant par la mort des contrevenants à l'ordre nazi-fasciste, c'est l'impossibilité de sortir de cet enfer qui se trouverait ainsi affirmé, témoignant peut-être alors du désespoir du réalisateur luimême. Mais, si l'on considère ces trois moments comme des actes de résistance en tant qu'ils se produisent de façon muette, et non pour eux-mêmes, alors une échappée devient envisageable. En effet, ce serait par le recours au silence qu'une trouée s'effectuerait, permettant d'échapper à cet univers, puisqu'au fond, c'est la parole en tant que telle qui se trouve compromise dans Salo, en ce que même les paroles de résistance parviennent à être récupérées par le fascisme. À travers le silence, c'est donc, paradoxalement, quelque chose de l'univers des "purs parlants" pasoliniens qui surgit, là où nous l'aurions, justement, le moins attendu. La pièce Orgie nous permet de confirmer que Pasolini considérait bien qu'une telle fonction pouvait être attribuée au silence: «Homme: Et que disaient les gens? / Femme: C'étaient les mêmes que chez toi. / Homme : Alors on ne parlait pas. / Femme : Oh si, les voix montaient de partout. / Le soleil frappait sur les gouttières bleues de sulfate? / Ou sur les murs de pierre au- 
dessus des fumiers? Et ces voix / rayaient de paix l'angoisse, tranquille, du jour. / Le crépuscule descendait doucement, comme une sorte de moût versé par les planètes / et son rouge était lourd comme une pâte divine ? / Eh bien ces voix étrangement joyeuses montaient, / du côté du clocher, ou des fermes sur la route asphaltée. / ET POURTANT PERSONNE NE PARLAIT. / [...] Homme : En vérité les miens étaient un peu différents. On vivait / dans une grande ville de province. [...] / ET POURTANT PERSONNE NE PARLAIT. [...] / Femme : Que les voix aient été / modulées dans un moyen âge rustique, passé / par le bon sens du XIX ${ }^{e}$ siècle, ou / dans une langue (une langue mal assimilée) / sur ce point il n'y a pas de doute: PERSONNE NE PARLAIT. / [...] Homme : Personne dans ce monde n'avait rien à dire à / quelqu'un d'autre : et pourtant tout résonnait de voix. / Et toi, comment as-tu appris à parler? / Femme : En écoutant ces voix. / Homme : Mais elles te disaient quelque chose? Quoi alors? / Femme : Oh non, ce n'était que des voix. Elles disaient / mon nom, c'est vrai, et montraient/ toutes les choses qui nous entouraient et dont nous nous servions / dans ce monde: MAIS ELLES NE PARLAIENT PAS / [...] Femme: Nous communiquions entre nous uniquement en faisant quelque chose. / Homme : Les Huns ou les Lombards avaient fait un petit temple / de la plus dure et la plus blanche des pierres; / l'Étrusque avait fait une tombe de tuf avec des sexes roses. / Femme : Ma mère faisait le poulet à la sauge, / et la tarte de farine jaune, sous la cendre. / [...] Homme: En nous apprenant à ne pas parler / voici ce qu'ils ont fait de nous. / Femme : Des personnes suffoquées par la joie de la honte $»^{23}$. Cette absence de parole apparaît donc avant tout comme une rupture vis-à-vis du langage instrumental, et comme un retour à la réalité, autant dire, par conséquent, à un univers plébéien, puisque le monde bourgeois est toujours caractérisé par Pasolini comme relevant de l'irréalité. Dans un tel monde où " personne ne parle ", la seule communication possible est action, et sous ce rapport, la dernière partie de Salo laisse le langage cinématographique assumer sa performativité spécifique, celle d'un langage d'action qui, par définition, en faisant, dit. Dans notre univers sursaturé de communication, où même l'éthique se veut communicationnelle, le saut par la fenêtre de la pianiste revêt une valeur de révélation, en ce qu'il initie un pur anachronisme, faisant surgir dans l'univers de Salo, surchargé de paroles et de commentaires littéraires croisés, ce qui lui est le plus contraire, l'innocent langage d'action propre à un univers précapitaliste autant dire que ce moment muet du film ressuscite un monde englouti. Le cinéma provoque ainsi l'émergence d'une image dialectique pleinement cinématographique: c'est en faisant se télescoper le cinéma contemporain de 1975 avec l'ancien cinéma muet, que Salo parvient, de façon immanente, à faire surgir une extériorité du sein même de l'autoréférentialité cinématographique, transperçant elle-même l'autoréférentialité culturelle et bavarde de la bande-son.

10 Ainsi, par-delà un cinéma de prose et un cinéma de poésie, on peut envisager que Pasolini expérimentait, à travers Salo ou les cent vingt journées de Sodome un cinéma d' action, très proche d'un cinéma muet, mais non par retour à une forme passée : là où le cinéma des origines était muet par nécessité, celui qu'inaugurait peut-être Pasolini, dans ce qui restera son ultime film, n'était que partiellement muet, et surtout ne l'était que par décision délibérée, c'est-à-dire sans réaliser le moindre pas en arrière du point de vue technique. C'est en cela que cette dernière forme cinématographique consonerait encore profondément avec les conceptions benjaminiennes, telles qu'on peut les appréhender, notamment du point de vue de la question du langage : parlant de la langue adamique, Benjamin se pose une question: "si la lampe, la montagne, le renard ne se communiquaient à l'homme, comment alors pourrait-il les nommer?»; et 
il en dégage cette réponse : «[...] il les nomme; il se communique en les nommant ${ }^{24}$. Or, du langage adamique des "noms", nous serions tombés dans un langage instrumental de simples «mots", incapables de nommer les choses en vérité, mais ce n'est pas là, pour Benjamin, une raison suffisante pour renoncer à entendre quelque chose d'un écho de la langue des origines, précisément à partir du lieu qui est le nôtre aujourd'hui, et non pas en cherchant à renouer de façon nostalgique avec le langage des origines. Le dernier film de Pasolini recueille ainsi quelques échos de la langue cinématographique des origines, sans renoncer pour autant à l'actualité de son cinéma - là est sa puissance intempestive.

\section{NOTES}

1. Walter Benjamin, cité par J. M. Monnoyer in Walter Benjamin, Écrits français, Gallimard, Paris 1991, p. 201.

2. Pier Paolo Pasolini, L'expérience hérétique, Payot, Paris 1976, p. 228.

3. Ibid., p. 205.

4. Ibid., p. 203.

5. Ibid., p. 228.

6. Ibid.

7. Ibid.

8. Ibid.

9. Ibid., p. 153.

10. Ibid.

11. Ibid.

12. Ibid., p. 153-154.

13. Ibid., p. 153 (c'est moi qui souligne).

14. Ibid.

15. W. Benjamin, Rastelli raconte... et autres récits, Seuil, Paris, 1971, p. 61.

16. P. P. Pasolini, Dialogues en public, Sorbier, Paris, 1980, p. 153.

17. P. P. Pasolini, L'expérience hérétique, p. 196.

18. W. Benjamin, Euvres III, Gallimard, Paris, 2000, p. 80-81.

19. P. P. Pasolini, L'expérience hérétique, p. 87.

20. Ibid., p. 140.

21. P. P. Pasolini, Pétrole, Gallimard, Paris, 1995, p. 357.

22. Gilles Deleuze, L'image-temps, Minuit, Paris, 1985, p. 228.

23. P. P. Pasolini, Théâtre, Actes Sud, Arles, 1995, p. 398-402.

24. W. Benjamin, Euvres I, Gallimard, Paris, 2000, p. 147. 